\title{
Knee and hip intra-articular adipose tissues (IAATs) compared to autologous subcutaneous adipose tissue: a specific phenotype for a central player in osteoarthritis
}

Florent Eymard ${ }^{1,2}$, Audrey Pigenet ${ }^{1}$, Danièle Citadelle ${ }^{1}$, Joan Tordjman ${ }^{3,4}$, Louise Foucher ${ }^{1}$, Cindy Rose $^{1}$, Charles-Henri Flouzat Lachaniette ${ }^{5}$, Christine Rouault ${ }^{3,4}$, Karine Clément ${ }^{3,4}$, Francis Berenbaum $^{1,2 * 6}$, Xavier Chevalier ${ }^{2}$ and Xavier Houard ${ }^{1}$

1 Sorbonne University, UPMC Univ Paris 06, INSERM, Centre de Recherche Saint-Antoine (CRSA), F-75012, Paris, France.

${ }^{2}$ Department of Rheumatology, AP-HP Henri Mondor Hospital, F-94010, Créteil Cedex, France.

${ }^{3}$ Sorbonne University, UPMC Univ Paris 06, INSERM UMR_S1166, Pitié-Salpêtrière Hospital, Paris, France.

4 Institute of Cardiometabolism and Nutrition, Assistance Publique-Hôpitaux de Paris, PitiéSalpêtrière Hospital, Paris, France.

5 Department of Orthopedic Surgery, AP-HP Henri Mondor Hospital, F-94010, Créteil Cedex, France.

${ }^{6}$ Department of Rheumatology, Inflammation-Immunopathology-Biotherapy Department (DHU i2B), AP-HP Saint-Antoine Hospital, F-75012 Paris, France.

*Address for correspondence:

Francis Berenbaum

INSERM UMR-S 938

"Metabolism and Age-related joint diseases"

Saint Antoine research center

27 rue Chaligny

F-75571 Cedex 12, Paris, France

francis.berenbaum@aphp.fr

Running title: intra-articular adipose tissues phenotyping

Word count: 3348

Authors received a financial support from the "Société Française de Rhumatologie" 


\begin{abstract}
Objectives. As compared to subcutaneous adipose tissue (SCAT), infrapatellar fat pad (IFP), the main knee intra-articular adipose tissue (IAAT), has an inflammatory phenotype in patients with osteoarthritis (OA). We phenotyped suprapatellar fat pad (SPFP) and hip acetabular fat pad (AFP), two other IAATs, to determinate the unique signature of IAATs compared to SCAT.
\end{abstract}

Methods. IFP, SPFP, AFP and autologous SCAT were obtained from OA patients during total knee $(n=38)$ or hip replacement $(n=5)$. Fibrosis and adipocyte area were analyzed by histology and vascularization, leukocyte and mast cell infiltration were analyzed by immunohistochemistry for von Willebrand factor, leukocytes and tryptase, respectively. Secretion of IL-6, IL-8 and PGE 2 was assessed by ELISA. The mRNA expression of adipocyte-associated genes (ATGL, LPL, PPAR- $\gamma$, FABP4 and CD36) and developmental genes (SFRP2, HoxC9 and EN1) was determined. The inflammatory response of isolated fibroblast-like synoviocytes (FLS) to autologous IFP and SPFP conditioned media was examined.

Results. Fibrosis, vascularization, and leukocyte and mast cell infiltration were greater in IAATs than SCAT, and levels of IL-6, IL-8 and PGE 2 were greater in all IAATs than SCAT. IFP and SPFP induced a similar inflammatory response to FLS. Adipocyte area was smaller in IAATs than SCAT. Adipocyte-associated and developmental genes showed a similar gene expression pattern in all IAATs, different from SCAT.

Conclusions. IFP but also SPFP and AFP (gathered under the term "IAAT") may play a deleterious role in OA by affecting joint homeostasis because of their inflammatory phenotype and their close interaction with synovium in the same functional unit.

Keywords: osteoarthritis, intra-articular adipose tissue, knee, hip, infrapatellar fat pad 


\section{Introduction}

Osteoarthritis (OA) is a whole-joint disease mainly characterized by progressive cartilage disappearance, subchondral bone remodeling and synovitis, which all act in concert for OA progression. In this context, many studies have focused on cartilage/subchondral bone crosstalk. Cartilage and subchondral bone are indeed now considered a unique functional unit. [1,2] Although synovitis was thought to mainly result from cartilage breakdown, several data suggest that it could be also involved in early stages of OA even before cartilage damage. [3, 4] Moreover, we and others have shown that knee OA synovitis may also depend on the release of inflammatory factors by the infrapatellar fat pad (IFP), located at the posterior surface of synovium. [5, 6]

IFP is one intra-articular adipose tissue (IAAT), which has received much attention for several years. IFP from knee OA patients releases many inflammatory factors in higher amounts as compared to autologous subcutaneous adipose tissue (SCAT). [6-8] IFP volume [9] and release of tumor necrosis factor $\alpha$ (TNF- $\alpha$ ) by IFP [8] are both positively associated with the body mass index of human OA patients. Similarly, in mice fed a high fat diet, weight gain and IFP volume were correlated with development of OA. [10] Positive associations were also found between adipocyte area and vascular infiltrates of IFP. [10] Consequently, IFP may have a paracrine function in other joint tissues in OA, especially the adjacent synovium. [5, 6]

In the best of our knowledge, only IFP has been studied in OA, although anatomically, other IAATs are indeed present in the joints. The suprapatellar fat pad (SPFP) is composed of the quadriceps fat pad and the pre-femoral fat pad, which are located above the patella and behind the suprapatellar bursa respectively (Supplementary Fig. 1). The posterior fat pad is in close contact with the posterior articular capsule behind the menisci. [11] Whether IFP has unique properties among IAATs is currently unknown. In addition, IAATs are not restricted to the knee. For instance, the coxofemoral joint contains one IAAT located in acetabular fossa and surrounding the 
ligamentum teres (acetabular fat pad, AFP) (Supplementary Fig. 1). Nothing is known about AFP in the context of arthritis. Whether IAATs from different joints share similar properties is unknown.

We investigated whether all IAATs acquire an inflammatory phenotype in OA like IFP, belonging to the same and unique type of adipose tissue (AT), which thus may act with the adjacent synovium as a unique functional unit. We used histological, molecular and functional characterization of IFP, SPFP and SCAT from autologous OA patients. In parallel, OA AFP and autologous SCAT were characterized. Fibrosis, vascular density, inflammatory infiltrates and adipocyte size were determined, as was the expression of inflammatory factors and molecules important for adipocyte function. The effect of different knee IAATs on fibroblast-like synoviocyte (FLS) inflammatory response was compared. 


\section{Materials and Methods}

\section{Adipose tissue and synovium samples}

Tissues were harvested from patients with end-stage symptomatic knee $(n=38)$ or hip OA $(n=5)$ undergoing surgery for total knee or hip replacement at Henri Mondor Hospital (Créteil, France). Sequential patients from whom informed consent was obtained were included. Surgeons harvested the IFP with the synovial membrane lining its posterior surface and the SPFP during knee replacement or AFP located in the bottom of acetabular fossa during hip replacement. The SCAT was harvested immediately below the scar. Tissues were stored in Dulbecco's modified Eagle's medium (DMEM) with $1 \%$ bovine serum albumin (BSA). The study was approved by the ethics committee of Henri Mondor Hospital and by the Assistance Publique Hôpitaux de Paris (approval no. 07-34) for biologic sample collection.

Omental and autologous subcutaneous adipose tissues were harvested from patients $(n=15$; female, n=14) operated at Visceral Surgery Department of Ambroise Paré Hospital (BoulogneBillancourt, France) for gastric banding $(n=2)$, gastric bypass $(n=7)$ or sleeve gastrectomy $(n=6)$. Mean age and mean BMI were $39.7 \pm 3.8$ (18-62) and 45.6 \pm 1.4 (36.4-53.7), respectively. All subjects are part of the BAR-ICAN study (study of obese subjects in bariatric surgery programs) (Ethical committee $\mathrm{N}^{\circ}$ 2014-april-13533).

\section{Generation of AT-conditioned medium}

ATs were carefully dissected as described. [6] For the IAAT samples, special care was taken to separate AT from the synovium. The absence of remaining synovium was checked on several samples by histology. Nevertheless, we cannot exclude the marginal presence of remaining synovium in some cases. To generate the AT conditioned medium, $300 \mathrm{mg}$ AT, minced into small pieces, was incubated in $1 \mathrm{~mL}$ of DMEM, $12.5 \mathrm{mM}$ glucose, and $1 \% \mathrm{BSA}$ for $1 \mathrm{hr}$ at $37^{\circ} \mathrm{C}$ in a humidified atmosphere of 5\% CO2/95\% air. The medium was removed and tissues were incubated 
in $1 \mathrm{~mL}$ of the same medium for $3 \mathrm{hr}$. Thereafter, conditioned media and tissues were collected separately, spun and frozen at $-80^{\circ} \mathrm{C}$.

\section{Isolation, culture and stimulation of FLS}

As described, [6] synovium was digested in $0.75 \mathrm{mg} / \mathrm{mL}$ collagenase/dispase and 0.075 mg/mL DNase (both from Roche Diagnostics) in FLS growth medium (RPMI 1640 Glutamax, 100 units/mL penicillin, $0.1 \mathrm{mg} / \mathrm{mL}$ streptomycin, $10 \mathrm{mM}$ HEPES, $2 \mathrm{mM}$ sodium pyruvate, $10 \%$ fetal calf serum) before seeding cells in culture plates. FLS were cultured at $37^{\circ} \mathrm{C}$ in a humidified atmosphere of $5 \% \mathrm{CO} 2 / 95 \%$ air.

FLS from passage 3 ( $n=6$ patients) were seeded at $10^{5}$ cells/well in 6 -well culture plates. Confluent FLS were left in depletion medium (growth medium without serum) for $24 \mathrm{hr}$ before washing with PBS and stimulation with IFP- or SPFP-conditioned medium (150 $\mu \mathrm{L})$ in depletion medium $(850 \mu \mathrm{L})$. Control FLS were incubated in depletion medium (850 $\mu \mathrm{L})$ with $150 \mu \mathrm{L}$ of medium used to generate tissue conditioned media. After 24-hr stimulation, FLS were rinsed twice with PBS and incubated in depletion medium for $24 \mathrm{hr}$. Conditioned media were kept, spun and stored at $-80^{\circ} \mathrm{C}$.

\section{Isolation of adipocytes}

IFP and SCAT were digested in $1 \mathrm{mg} / \mathrm{mL}$ collagenase (Roche Diagnostics) in DMEM 4.5 g/L glucose, 100 units/mL penicillin, $0.1 \mathrm{mg} / \mathrm{mL}$ streptomycin, $15 \mathrm{mM}$ HEPES, $0.2 \%$ BSA for $1 \mathrm{hr}$ at $37^{\circ} \mathrm{C}$. The adipose suspension was then filtered through a 100 micron mesh and centrifuged for 6 min at $150 \mathrm{~g}$. The upper phase containing adipocytes was separated and washed 2 times with PBS. After a final centrifugation (6 min at $150 \mathrm{~g}$ ), adipocytes were lysed for gene expression analysis. 


\section{Total RNA extraction and quantitative RT-PCR}

Total RNA was isolated by using the Reliaprep RNA Cell miniprep system (Promega). RNA (250-1,000 ng) was reverse transcribed by using the Omniscript RT kit (Qiagen). Gene expression was analyzed by quantitative RT-PCR with Roche Diagnostics LightCycler 480 in a $12-\mu \mathrm{L}$ final volume with specific primers $(10 \mu \mathrm{M})$ (Supplementary Table 1) and GoTaq PCR Master Mix (Promega). PCR amplification involved a denaturation step ( 5 min at $95^{\circ} \mathrm{C}$ ) followed by 40 cycles of $10 \mathrm{sec}$ at $95^{\circ} \mathrm{C}, 15 \mathrm{sec}$ at $60^{\circ} \mathrm{C}$, and $10 \mathrm{sec}$ at $72^{\circ} \mathrm{C}$.

For each PCR, cDNA was run in duplicate in parallel with serial dilutions of a cDNA mixture tested for each primer pair to generate a standard linear curve, which was used to estimate the amplification efficiency. The relative mRNA expression for all genes analyzed was normalized to that of 18S RNA (used as the internal reference gene) and determined by using the efficiency method with Light Cycler 480 software.

\section{Enzyme-linked immunosorbent assay (ELISA)}

ELISA kits were used to determine the concentrations of interleukin 6 (IL-6), IL-8 (both from Sanquin-PeliKine), prostaglandin $\mathrm{E}_{2}\left(\mathrm{PGE}_{2}\right.$; Cayman Chemical), matrix metalloproteinase 1 (MMP-1; from R\&D Systems) in AT and/or FLS-conditioned medium.

\section{Histology and immunohistochemistry study}

AT samples were fixed in $3.7 \%$ paraformaldehyde, embedded in paraffin and serially sectioned $(5 \mu \mathrm{m})$. Sections were stained with picrosirius red (Sigma). Immunohistochemistry involved mouse monoclonal antibodies to CD45 (leukocytes, clone 2B11+PD7/26, Dako) (dilution 1:100), tryptase (mast cells, AA1, Santa Cruz Biotechnology) (dilution 1:100), CD3 (Tlymphocytes, clone F7.2.38, Dako) (dilution 1:50), CD20 (B-lymphocytes, clone L26, Dako) (dilution 1:100) and CD68 (macrophages, clone PG-M1, Dako) (dilution 1:100) and von Willbrand factor (vWF) (endothelial cells, clone F8/86, Dako) (dilution 1:500) as primary antibodies. For all 
antibodies except vWF, the R.T.U Vectastain kit (Vector) was used for detection followed by counterstaining with Mayer's hematoxylin. Immunofluorescent detection of vWF involved horseradish peroxidaseconjugated secondary rabbit anti-mouse IgG antibody (Abliance) and the TSA Plus Cyanine 3 System (Perkin Elmer). Irrelevant control antibodies (Dako) were incubated at the same concentration to assess non-specific staining.

\section{Morphometric analysis}

Sections stained with picrosirius red were used for fibrosis quantification and adipocyte area determination. Digital images of magnification views (x20) of tissue sections were captured by using an Olympus DP73 camera (Olympus) on an Olympus BX43 microscope. Fibrosis analysis involved histomorphometry with CaloPix software (Chatillon, France) with content color thresholds. The quantification of total fibrosis was expressed as the ratio of fibrous tissue area stained with picrosirius red/total tissue surface. [12] For adipocyte mean area determination, two independent observers blindly measured the area of 40 adipocytes located in the center of the tissue section and values were averaged. Vessel number and vascular density were quantified after vWF immunostaining. The number of vessels was measured in the whole section and normalized to the tissue area. Vascular density was quantified as the proportion of vWF-positive area normalized to tissue area. Infiltration of CD45- and tryptase-positive cells within the tissue area was graded as 0 , no or sparse positive cells; 1 , several positive cells; and 2, numerous positive cells with several clusters.

\section{Statistical analysis}

Paired Wilcoxon non-parametric rank test (Statview software, version 4.57 SAS Inc.) was used for analysis. Data are presented as mean \pm SD. $\mathrm{P}<0.05$ was considered statistically significant. 


\section{Results}

\section{Patient characteristics}

We included patients with severe and symptomatic knee $(n=38)$ and hip OA $(n=5)$. Characteristics of each group are in Supplementary Table 2. In the knee OA group, the mean age was $73.3 \pm 6.5$ years and $29(76.3 \%)$ were women. The mean BMI was $29.3 \pm 5.0 \mathrm{~kg} / \mathrm{m}^{2}$. In the hip OA group, the mean age was $73.0 \pm 3.3$ years and $3(60.0 \%)$ were women. The mean BMI was $27.4 \pm 4.4 \mathrm{~kg} / \mathrm{m}^{2}$.

\section{Fibrosis and inflammatory infiltration of $\mathrm{OA}$ intra-articular adipose tissues}

At the time of dissection, all harvested IAATs, whatever their knee or hip origin, had more fibrous tissue than autologous SCAT. This observation was confirmed at histological level after quantification of picrosirius red staining (Fig. 1). In knee OA, fibrosis in IFP and SPFP accounted for $30.9 \pm 18.6 \%$ and $26.9 \pm 9.4 \%$ of tissue area, respectively, and was significantly more extended than in SCAT (18.9 $\pm 8.5 \%$ of tissue area, $\mathrm{p}=0.028$ and $\mathrm{p}=0.028$, respectively). This difference was even more pronounced between AFP and corresponding SCAT $(69.2 \pm 9.5 \%$ and $21.3 \pm 6.0 \%$ of tissue area, respectively) (Fig. 1B and C). Given the small number of patients, no statistical analysis was performed for hip tissue. In IAATs, fibrous tissue accumulated between adipocyte lobules as large fascicles. In some cases, adipocytes and fibers were intermingled (Fig. 1A panels a and b). In contrast, the fibrous part of SCAT surrounded adipocyte lobules and few fibers were detected among clusters of adipocytes (Fig. 1A panel c). The vascular network also appeared different between IAATs and SCAT. Vessel number to tissue area was significantly increased 2.2-fold between both IFP and SPFP and SCAT (data not shown). The vascular area to tissue area was significantly greater in IFP and SPFP than SCAT ( $p=0.003$ and $p=0.006$, respectively) (Fig. 1D), with no difference between IFP and SPFP. As observed in knee IAATs, a higher vessel number and vascular area to tissue area were quantified in AFP compared to autologous SCAT (Fig. 1E). The 
fibrous part of ATs contained more vessels, which were also larger than within adipocyte lobules. Tissue fibrosis and vascularization were associated neither with the obese/non-obese status of the patients nor with gender, with the exception of a lower vascular area, in females only (Supplementary Table 3). In addition to fibrosis and vascularization, CD45- and tryptase-positive cell infiltration was greater in knee and hip IAATs than autologous SCAT (Fig. 2). Leukocytes and mast cells preferentially accumulated in perivascular areas in fibrous parts of ATs than in adipocyte lobules. Inflammatory infiltrates consisted mainly of macrophages. Some T- and B-lymphocytes could also be observed in IAATs (Supplementary Fig. 2).

\section{Common inflammatory secretion pattern of IAATs}

As we previously reported, [6, 7] IFP from OA patients produced significantly more IL-6, IL-8 and PGE 2 than autologous SCAT (Fig. 3A-C). Similarly, SPFP also secreted higher amounts of these inflammatory mediators as compared to SCAT. The release was 6.2-, 6.7- and 189.1-fold higher by SPFP than SCAT for IL-6 $(\mathrm{p}<0.0001)$, IL-8 $(\mathrm{p}=0.002)$ and PGE $(\mathrm{p}<0.0001)$, respectively. No significant difference was found between IFP and SPFP. AFP also secreted significantly more inflammatory factors than autologous SCAT (Fig. 3D-E). No relationship was found between obesity and any of the inflammatory factors. We only found a lower release of $\mathrm{PGE}_{2}$ by SCAT in females only (Supplementary Table 3).

As a consequence of the inflammatory pattern of IAAT secretion products, both IFP- and SPFP conditioned media induced an inflammatory and prodegradative response to autologous FLS (Fig. 4). The production of IL-6 and MMP-1 was indeed strongly stimulated by IFP (8.6-fold, p=0.028, and 3.7-fold, $\mathrm{p}=0.028$, respectively) and SPFP-conditioned media (15.6-fold, $\mathrm{p}=0.046$, and 3.8-fold, $\mathrm{p}=0.043$, respectively), as compared to unstimulated control cells. 


\section{Specific phenotype of IAAT-derived adipocytes}

Morphometric analysis of adipocytes revealed a smaller size of adipocytes within IAATs than SCAT. IFP and SPFP adipocyte mean area was 0.6 -fold $(\mathrm{p}=0.002)$ and 0.7 -fold smaller $(\mathrm{p}=0.007)$, respectively, than that from autologous SCAT (Fig. 5). IFP and SPFP did not differ in adipocyte mean area. As observed in knee IAATs, the mean surface of AFP-derived adipocytes was 0.7-fold smaller than that from autologous SCAT (Fig. 5). No relationship was found between obesity or gender with the adipocyte area of knee IAATs and SCAT (Supplementary Table 3). This result suggests that adipocytes present within IAATs and SCAT have distinct phenotypes.

EN1, SFRP2, HoxC9 and Wt1 are genes that are differentially expressed by SCAT and intra-abdominal AT and their isolated adipocytes. ([13-15] and supplementary Fig. 3) Here, IAATs and SCAT also differentially expressed EN1 mRNA, with an expression strongly decreased in all IAATs as compared to SCAT (IFP: 0.3-fold, $\mathrm{p}=0.006$, SPFP: 0.2 -fold, $\mathrm{p}=0.006$, and AFP: 0.3 -fold, $\mathrm{p}=0.046$ ) (Fig. 6H). Similarly, the mRNA level of HoxC9 was lower in SPFP and AFP than autologous SCAT (SPFP: 0.6-fold, $\mathrm{p}=0.025$, and AFP: 0.3-fold, $\mathrm{p}=0.043$ ). IFP and SCAT did not differ in HoxC9 expression and all ATs showed a similar mRNA expression of SFRP2 (Fig. 6). Wt1 was not expressed by IAATs.

PPAR- $\gamma$, CD36, FABP4, LPL and ATGL are involved in adipogenesis, intracellular fatty acid transfer and trafficking, lipogenesis and lipolysis. They were also differentially expressed in IAATs and SCAT (Fig. 6). The mRNA expression of CD36, FABP4, LPL and ATGL was significantly decreased in IFP, SPFP and AFP as compared to the autologous SCAT. PPAR- $\gamma$ mRNA expression was lower in SPFP and AFP than SCAT. In contrast, PPAR- $\gamma$ mRNA expression was similar in IFP and SCAT. Interestingly, some differences were observed between IFP and SPFP. The mRNA levels of PPAR- $\gamma(\mathrm{p}=0.002)$ and CD36 $(\mathrm{p}=0.007)$ were lower in SPFP than autologous IFP.

To exclude that differences in gene expression between IAATs and SCAT could be due to the accumulation of fibrosis within IAATs and thus to a lower proportion of adipocytes within the 
whole AT, we analyzed the expression of ATGL, LPL and CD36 in isolated adipocytes from IFP and SCAT (Fig. 6I). As observed in whole AT, the mRNA expression of ATGL, LPL and CD36 was lower in IFP- than SCAT-derived adipocytes. 


\section{Discussion}

In recent years, IFP has received much attention for its possible involvement in OA. IFP volume or surface is modified in OA and could be associated with structural damage and pain. [16] IFPs from early- and end-stage OA display a different gene expression pattern. [17] OA IFP also shows an inflammatory phenotype characterized by a higher expression and secretion of inflammatory factors than autologous SCAT. [6, 7] Consistently, IFP stimulates an inflammatory response to FLS, $[5,6]$ which suggests that the functional interaction of IFP with synovium may be a mechanism of inducing OA synovitis. Of note, several IAATs are present within the knee and IAATs are not restricted to the knee. In this study, we wondered whether IFP is unique among IAATs or whether all IAATs share common properties. Interestingly, all IAATs feature a similar histological pattern: increased fibrosis, vascularization and leukocyte infiltration, as compared to autologous SCAT. They express and secrete a higher level of inflammatory factors (IL-6, IL-8 and PGE2). All these features were independent of gender or BMI of patients. Like IFP, SPFP induces an inflammatory response to FLS. All IAATs and isolated adipocytes express a lower level of genes associated with adipocyte function. The size of adipocytes is lower in IAATs than SCAT. These results suggest that all IAATs belong to the same type of AT and support that IAATs and their adjacent synovium should be considered a unique functional unit.

Our results show that IAATs differ from SCAT. Although we cannot exclude that the proportion of fibrous and vascular tissue may explain some molecular differences we observed between IAATs and SCAT, the distinct gene expression pattern of isolated adipocytes from IFP and SCAT argue for a peculiar IAAT phenotype. Interestingly, IAATs seem to share similar properties with visceral AT (VAT), the other main type of white ATs. Indeed, the size of adipocytes from omental or perivascular AT are smaller than in SCAT depots, $[18,19]$ as we found for IAATs. Similarly, the level of adipocyte-associated genes is reduced in adipocytes from perivascular and perirenal ATs. [18] SCAT and VATs differentially express developmental genes, including EN1 
and HoxC9, whose expression is decreased in intra-abdominal VAT, whereas the expression of SFRP2 is increased. ([14, 15] and data not shown) Our results also show lower levels of both EN1 and HoxC9 in IAATs than SCAT. Similarly, some developmental genes, including EN1, were differentially expressed by perivascular AT and SCAT, with no difference found between SCAT and perirenal AT. [18] This latter observation highlights intrinsic differences between VATs. [15] Nevertheless, Chau et al. recently showed that VATs from six different depots but not SCAT all express Wt-1. [13] Interestingly, in our study, no expression of Wt-1 was observed in IAATs, whereas it was in omental VAT. This suggests that IAATs may be a specific AT different from SCAT and VAT while sharing several common properties with VATs.

OA IAATs are characterized by a higher fibrotic index, inflammatory infiltrates and vascularization than autologous SCAT. Increased leukocyte infiltration and mast cell number in OA IFP has been reported. [8] Leukocytes and mast cells preferentially localize within fibrotic areas. Similar observations were reported in omental VAT from obese patients. [12] Inflammation stimulates fibrosis in AT, and macrophages can express extracellular matrix components. [20] Of note, monoiodoacetate injection can induce OA and provoke IFP inflammation and fibrosis. [21, 22] IFP area and vascularization are increased in the murine high-fat diet-induced OA model. [10] In addition to inflammation, AT fibrosis can be induced by mechanical stimulus, [23] which is relevant for knee and hip OA. According to the link between fibrosis, inflammation and mechanical load, we suggest that the peculiar phenotype of IAATs we describe here could depend on intrinsic properties of IAATs. A recent study comparing the histological characteristics of IFP from cadavers without knee OA to autologous knee SCAT and heterologous abdominal SCAT [24] reported that IFP adipocytes were smaller than those from SCAT. However, their results on tissue fibrosis differ from ours. Indeed, interlobular septa of IFP were thinner than those from knee SCAT, whereas no quantitative difference of the intercellular space was observed. We have no explanation for this discrepancy between their study and ours. Nevertheless, we never observed SCAT as fibrous as they showed. The peculiar phenotype of IAATs may also be acquired during the course of OA. Indeed, Gandhi et al. showed differences in gene expression pattern between IFP from early and late OA patients. [17] 
To conclude, knee and hip OA IAATs share a common phenotype, including a less adipogenic profile but higher fibrotic and inflammatory characteristics than autologous SCAT. IAATs could be considered a subgroup of AT, such as visceral, muscular or perivascular AT. The IAAT impact on joint homeostasis could be related to its inflammatory and metabolic profile and mediated by close interactions with synovium in a same functional unit. IAATs may be new players in OA disease progression. 


\section{Conflict of interest}

None.

\section{Acknowledgments}

The authors thank the "Société Française de Rhumatologie" for financial support. Authors also thank Gilles Le Naour and the department of pathology of AP-HP Pitie Salpetriere Hospital (Paris, France).

\section{References}

1. Lories RJ, Luyten FP. The bone-cartilage unit in osteoarthritis. Nat Rev Rheumatol. 2010 Jan;7(1):43-9.

2. Mahjoub M, Berenbaum F, Houard X. Why subchondral bone in osteoarthritis? The importance of the cartilage bone interface in osteoarthritis. Osteoporos Int. 2012 Dec;23 Suppl 8:841-6.

3. Atukorala I, Kwoh CK, Guermazi A, Roemer FW, Boudreau RM, Hannon MJ, et al. Synovitis in knee osteoarthritis: a precursor of disease? Ann Rheum Dis. 2016 Feb;75(2):390-5.

4. Wang X, Blizzard L, Jin X, Chen Z, Zhu Z, Han W, et al. Quantitative Assessment of Knee Effusion-Synovitis in Older Adults: Association With Knee Structural Abnormalities. Arthritis Rheumatol. 2016 Apr;68(4):837-44.

5. Bastiaansen-Jenniskens YM, Wei W, Feijt C, Waarsing JH, Verhaar JA, Zuurmond AM, et al. Stimulation of fibrotic processes by the infrapatellar fat pad in cultured synoviocytes from patients with osteoarthritis: a possible role for prostaglandin f2alpha. Arthritis Rheum. 2013 Aug;65(8):2070-80.

6. Eymard F, Pigenet A, Citadelle D, Flouzat-Lachaniette CH, Poignard A, Benelli C, et al. Induction of an inflammatory and prodegradative phenotype in autologous fibroblast-like 
synoviocytes by the infrapatellar fat pad from patients with knee osteoarthritis. Arthritis Rheumatol. 2014 Aug;66(8):2165-74.

7. Distel E, Cadoudal T, Durant S, Poignard A, Chevalier X, Benelli C. The infrapatellar fat pad in knee osteoarthritis: an important source of interleukin-6 and its soluble receptor. Arthritis Rheum. 2009 Nov;60(11):3374-7.

8. Klein-Wieringa IR, Kloppenburg M, Bastiaansen-Jenniskens YM, Yusuf E, Kwekkeboom JC, El-Bannoudi $\mathrm{H}$, et al. The infrapatellar fat pad of patients with osteoarthritis has an inflammatory phenotype. Ann Rheum Dis. 2011 May;70(5):851-7.

9. Diepold J, Ruhdorfer A, Dannhauer T, Wirth W, Steidle E, Eckstein F. Sex-differences of the healthy infra-patellar (Hoffa) fat pad in relation to intermuscular and subcutaneous fat content-data from the Osteoarthritis Initiative. Ann Anat. 2015 Jul;200:30-6.

10. Iwata M, Ochi H, Hara Y, Tagawa M, Koga D, Okawa A, et al. Initial responses of articular tissues in a murine high-fat diet-induced osteoarthritis model: pivotal role of the IPFP as a cytokine fountain. PLoS One. 2013;8(4):e60706.

11. Gallagher J, Tierney P, Murray P, O'Brien M. The infrapatellar fat pad: anatomy and clinical correlations. Knee Surg Sports Traumatol Arthrosc. 2005 May;13(4):268-72.

12. Divoux A, Tordjman J, Lacasa D, Veyrie N, Hugol D, Aissat A, et al. Fibrosis in human adipose tissue: composition, distribution, and link with lipid metabolism and fat mass loss. Diabetes. 2010 Nov;59(11):2817-25.

13. Chau YY, Bandiera R, Serrels A, Martinez-Estrada OM, Qing W, Lee M, et al. Visceral and subcutaneous fat have different origins and evidence supports a mesothelial source. Nat Cell Biol. 2014 Apr;16(4):367-75.

14. Gesta S, Bluher M, Yamamoto Y, Norris AW, Berndt J, Kralisch S, et al. Evidence for a role of developmental genes in the origin of obesity and body fat distribution. Proc Natl Acad Sci U S A. 2006 Apr 25;103(17):6676-81. 
15. Tchkonia T, Lenburg M, Thomou T, Giorgadze N, Frampton G, Pirtskhalava T, et al. Identification of depot-specific human fat cell progenitors through distinct expression profiles and developmental gene patterns. Am J Physiol Endocrinol Metab. 2007 Jan;292(1):E298-307.

16. Eymard F, Chevalier X. Inflammation of the infrapatellar fat pad. Joint Bone Spine. 2016 Jul;83(4):389-93.

17. Gandhi R, Takahashi M, Virtanen C, Syed K, Davey JR, Mahomed NN. Microarray analysis of the infrapatellar fat pad in knee osteoarthritis: relationship with joint inflammation. J Rheumatol. 2011 Sep;38(9):1966-72.

18. Chatterjee TK, Stoll LL, Denning GM, Harrelson A, Blomkalns AL, Idelman G, et al. Proinflammatory phenotype of perivascular adipocytes: influence of high-fat feeding. Circ Res. 2009 Feb 27;104(4):541-9.

19. Tchernof A, Belanger C, Morisset AS, Richard C, Mailloux J, Laberge P, et al. Regional differences in adipose tissue metabolism in women: minor effect of obesity and body fat distribution. Diabetes. 2006 May;55(5):1353-60.

20. Sun K, Tordjman J, Clement K, Scherer PE. Fibrosis and adipose tissue dysfunction. Cell Metab. 2013 Oct 1;18(4):470-7.

21. Clements KM, Ball AD, Jones HB, Brinckmann S, Read SJ, Murray F. Cellular and histopathological changes in the infrapatellar fat pad in the monoiodoacetate model of osteoarthritis pain. Osteoarthritis Cartilage. 2009 Jun;17(6):805-12.

22. Udo M, Muneta T, Tsuji K, Ozeki N, Nakagawa Y, Ohara T, et al. Monoiodoacetic acid induces arthritis and synovitis in rats in a dose- and time-dependent manner: proposed modelspecific scoring systems. Osteoarthritis Cartilage. 2016 Jul;24(7):1284-91.

23. Pellegrinelli V, Heuvingh J, du Roure O, Rouault C, Devulder A, Klein C, et al. Human adipocyte function is impacted by mechanical cues. J Pathol. 2014 Jun;233(2):183-95.

24. Macchi V, Porzionato A, Sarasin G, Petrelli L, Guidolin D, Rossato M, et al. The Infrapatellar Adipose Body: A Histotopographic Study. Cells Tissues Organs. 2016;201(3):220-31. 


\section{Figure Legends}

Figure 1. Histological characterization of fibrosis and vascularization in intra-articular adipose tissue (IAAT) and subcutaneous adipose tissue (SCAT) from end-stage osteoarthritis (OA) patients.

A) Paraffin sections $(5 \mu \mathrm{m}$ ) of infrapatellar fat pad (IFP) (a and f), suprapatellar fat pad (SPFP) (b and g), acetabular fat pad (AFP) (d and i) and SCAT (c, e, h and j) (n=16 for knee and n=3 for hip tissues) were stained with picrosirius red for fibrosis determination (a-e) and for von Willebrand factor (vWF) (f-j) for vessel quantification. Digital images of magnification views (x4) of tissue sections were captured. In IAATs, fibrous tissue (stained in red) accumulated between adipocyte lobules as large fascicles. In some cases, adipocytes and fibers were intermingled. In contrast, the fibrous part of SCAT surrounded adipocyte lobules and few fibers were detected among clusters of adipocytes ( $c$ and e). Quantification of the proportion of fibrosis and ratio of vessel-to-tissue area in knee $(n=16)(B$ and C) and hip tissues $(n=3)(D$ and E). Knee IAATs have significantly greater percentage of fibrosis and vessel area than autologous SCAT. Similar observations are made for AFP compared to hip SCAT. Horizontal bar is mean and dots represent each patient. $* \mathrm{P}<0.05$

Figure 2. Histological characterization of leukocyte infiltration in IAAT and SCAT from endstage OA patients.

A) Paraffin sections (5 $\mu \mathrm{m}$ ) of IFP (a and f), SPFP (b and g), AFP (d and i) and SCAT (c, e, $h$ and $j$ ) (n=13 for knee and $n=3$ for hip tissues) were immunostained for CD45 leukocytes (a-e) and tryptase, which targets mast cells (f-j). CD45- and tryptase-positive cells were mainly observed in the fibrous part and in perivascular areas of the adipose tissue. Original magnification x20. B-E) 
Quantification of the leukocyte (B and D) and mast cell infiltrates (C and E) of knee (B and C) and hip IAATs and SCAT (D and E). More leukocytes and mast cells are present in IAATs than SCAT. Data are mean $\pm \mathrm{SD} . * \mathrm{P}<0.05$

Figure 3. Differential release of inflammatory factors by knee and hip IAATs and SCAT from end-stage OA patients.

Secretion of interleukin 6 (IL-6) (A and D), IL-8 (B and E) and prostaglandin E2 (PGE2) (C and F) by knee (A-C) and hip IAATs (D-F) and autologous SCAT in conditioned media. Measurements of 19 and 5 patients with knee and hip OA, respectively. Horizontal bar is mean and dots represent each patient. $* \mathrm{P}<0.05$

Figure 4. Inflammatory response of fibroblast-like synoviocytes (FLS) by stimulation by autologous knee intra-articular adipose tissues

FLS from 6 OA patients were treated or not with conditioned media from autologous IFP and SPFP. Secretion of IL-6 (A) and MMP-1 (B) in FLS-conditioned media was determined by ELISA. Horizontal bar is mean and dots represent each patient. $* \mathrm{P}<0.05$

Figure 5. Mean adipocyte size in IAATs and SCAT from end-stage OA patients.

A) Paraffin sections $(5 \mu \mathrm{m})$ of IFP (a), SPFP (b), AFP (d) and SCAT (c and e) (n=16 for knee and n=3 for hip tissues) were stained with picrosirius red and adipocyte size was quantified. B) Quantification of mean adipocyte size of knee and hip IAATs. The mean size of adipocytes from knee IAATs is significantly lower than those of autologous SCAT. A similar observation is made for AFP compared to hip SCAT. Data are expressed as ratio of mean adipocyte size between each 
IAAT and its corresponding SCAT (set at 1.0, horizontal dotted line). Horizontal bar is mean and dots represent each patient. $* \mathrm{P}<0.05$

Figure 6. Differential gene expression pattern of knee and hip IAATs and autologous SCAT from end-stage $\mathrm{OA}$ patients.

A-H) Relative mRNA expression of PPAR- $\gamma$ (A), ATGL (B), FABP4 (C), CD36 (D), LPL (E), SFRP2 (F) HoxC9 (G) and EN1 (H) by IFP, SPFP and AFP to their corresponding SCAT from knee $(\mathrm{n}=12)$ and hip ( $\mathrm{n}=5)$ OA patients. I) Adipocytes from IFP and SCAT were isolated from 4 patients before determining mRNA expression of ATGL, CD36 and LPL. Data are fold increase compared to SCAT, whose mRNA expression was set to 1 for each patient (horizontal dotted line). Horizontal bar is mean and dots represent each patient. ${ }^{*} \mathrm{P}<0.05$ vs SCAT and \#P<0.05 vs SPFP. 


\section{Supplementary Figure 1. Intra-articular fat pads of the knee and hip}

A) Several intra-articular fat pads are present in the knee. The largest is the infrapatellar fat pad (IFP) or Hoffa's tissue, which is located under the patella, behind the patellar tendon and in front of the femoral condyles and tibial plateau. The suprapatellar fat pad (SPFP) is composed of the quadriceps fat pad and the pre-femoral fat pad, which are located above the patella and behind the suprapatellar bursa, respectively. The posterior fat pad (PFP) is in close contact with the posterior articular capsule behind the menisci. B) The hip also contains one IAAT, the acetabular fat pad (AFP), located in the acetabular fossa and surrounding the ligamentum teres. Illustrations are from Servier Medical Art with modifications.

Supplementary Figure 2. Histological characterization of leukocyte infiltration in IAAT and SCAT from end-stage OA patients.

A) Paraffin sections $(5 \mu \mathrm{m})$ of IFP (a, f and j), SPFP (b, g and j), AFP (d, h and k) and SCAT (c and e, h) ( $\mathrm{n}=13$ for knee and $\mathrm{n}=3$ for hip tissues) were immunostained for CD68 macrophages (a-e), CD3 T-lymphocytes (f-h) and CD20 B-lymphocytes (i-k). Macrophages were present in IAATs and SCATs although found in higher number in IAATs from knee and hip. Some IAATs also contained T- and B-lymphocytes. Original magnification x20 (a-e, g and j) and x10 (f, $\mathrm{h}, \mathrm{i}$ and $\mathrm{k})$.

Supplementary Figure 3. Differential gene expression pattern of omental adipose tissue and autologous SCAT.

A-D) Relative mRNA expression of EN1 (A), HoxC9 (B), SFRP2 (C) and Wt-1 (D) by omental (OM) adipose tissue and autologous SCAT $(n=15)$. Horizontal bar is mean and dots represent each patient. $* \mathrm{P}<0.05$. 
A

Knee

Hip
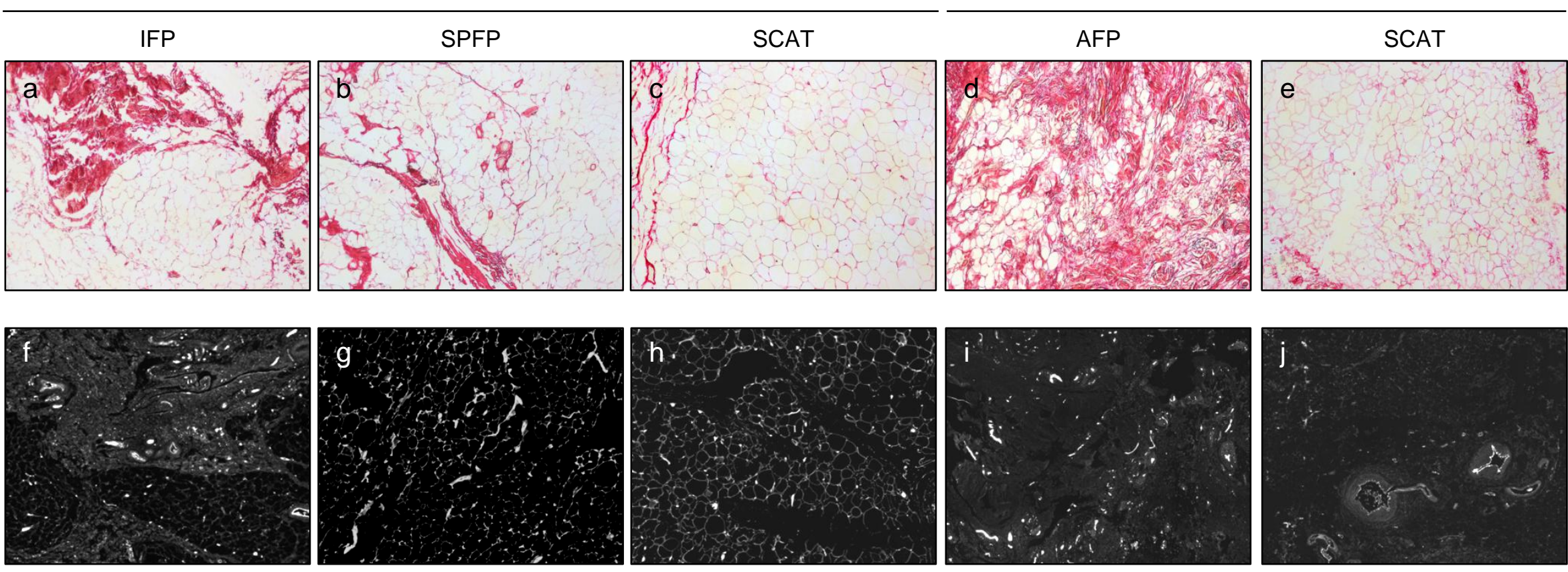

B

C
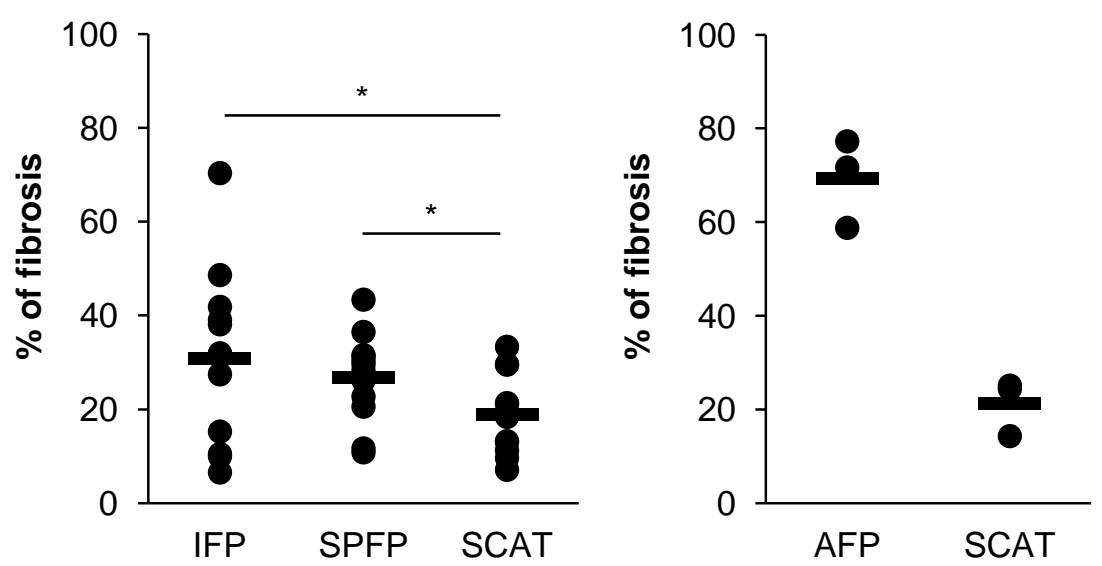

D

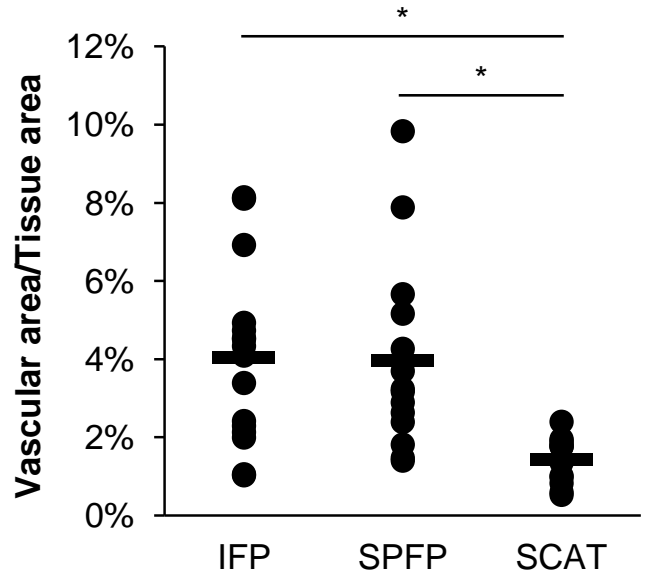

E

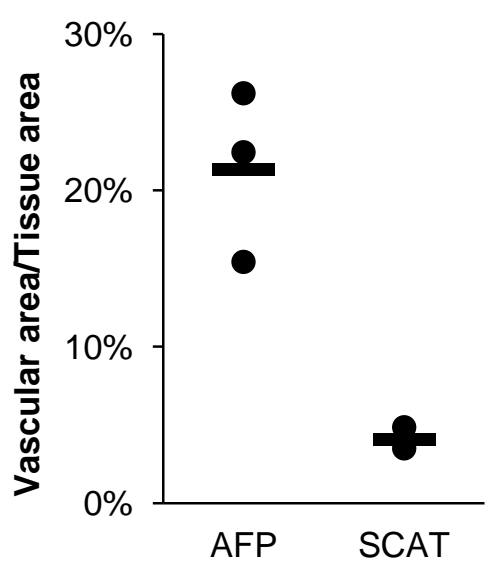

Figure 1 
A

Knee

Hip
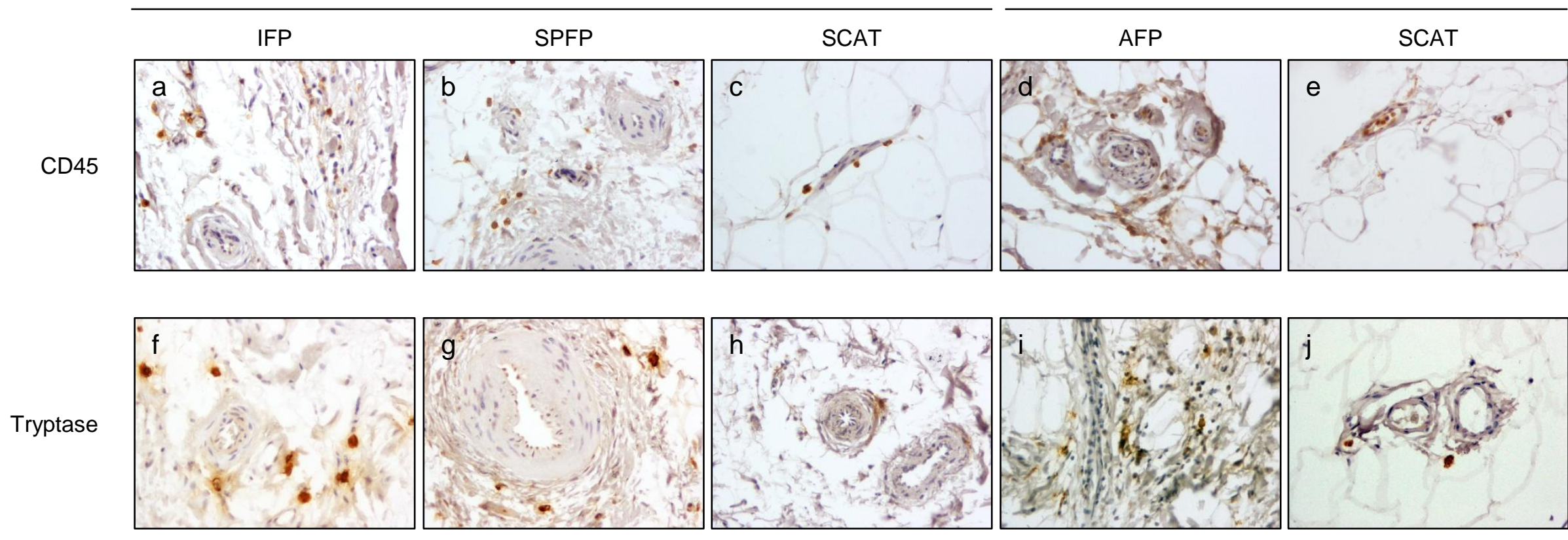

B

C

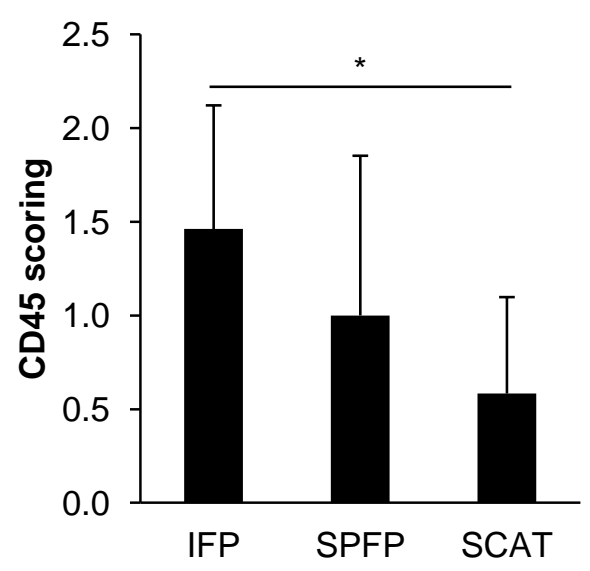

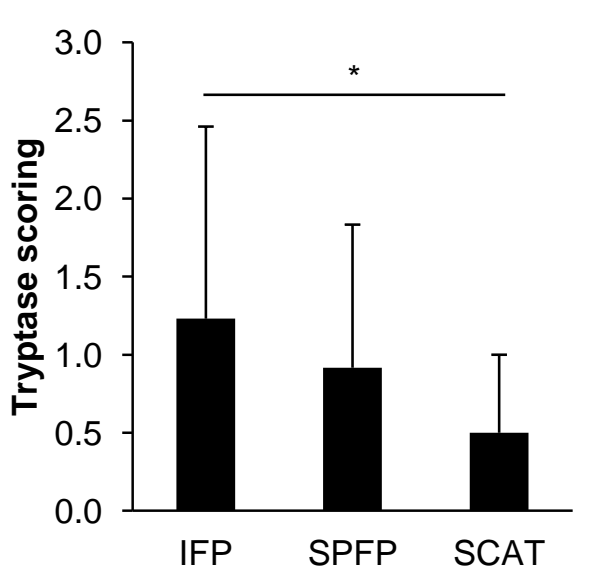

D

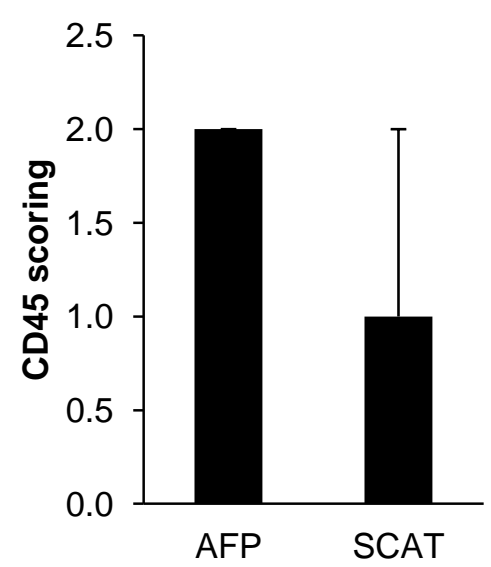

E

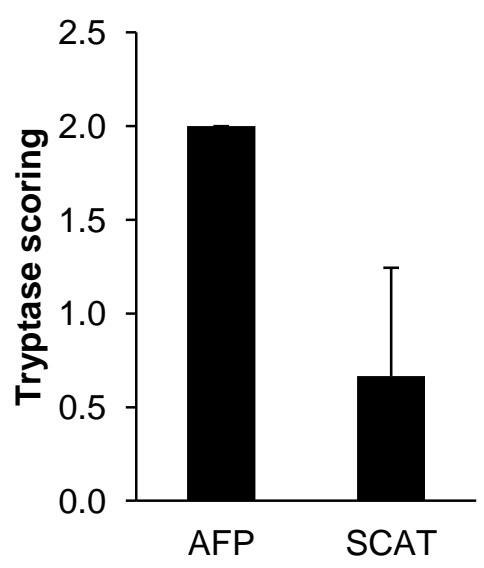

Figure 2 
A

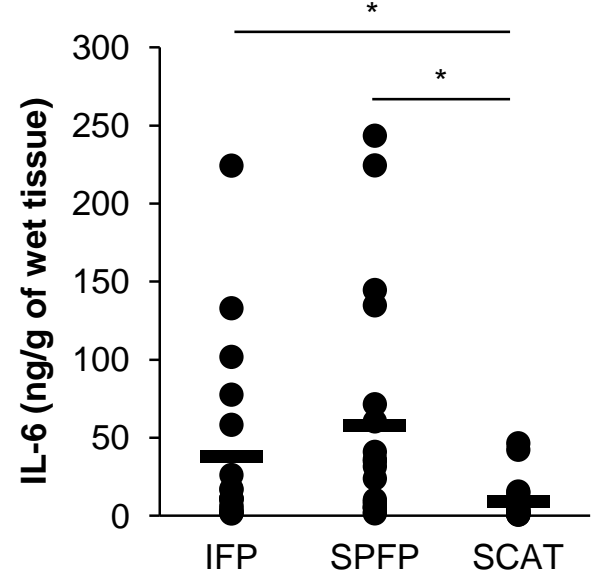

D

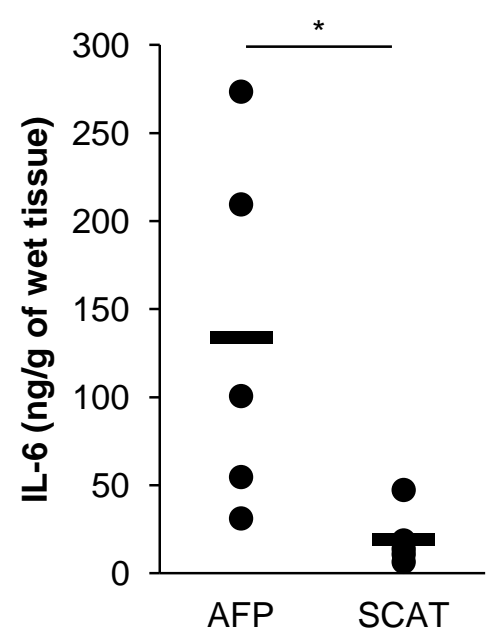

B

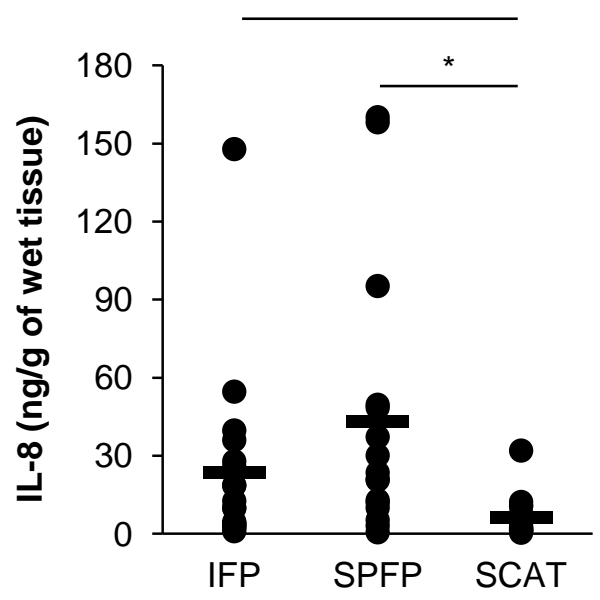

E

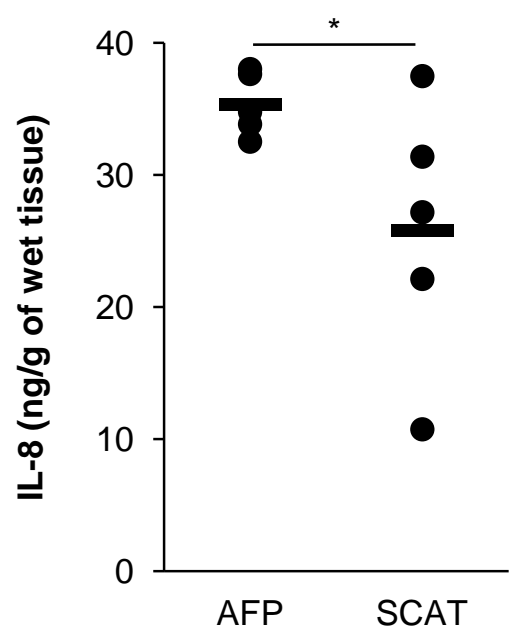

C

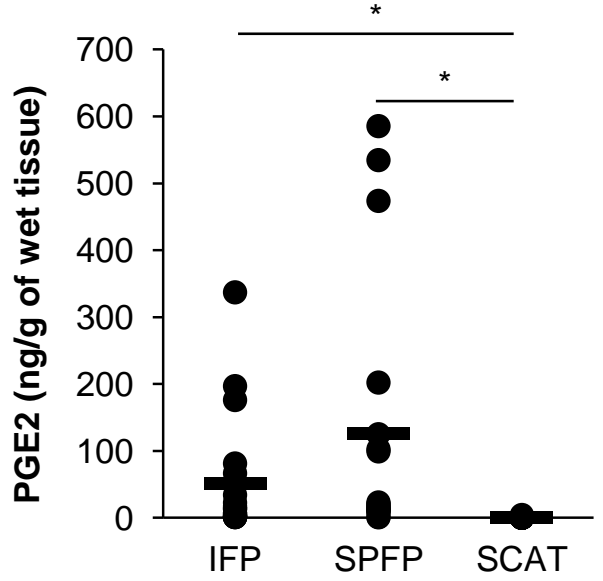

F

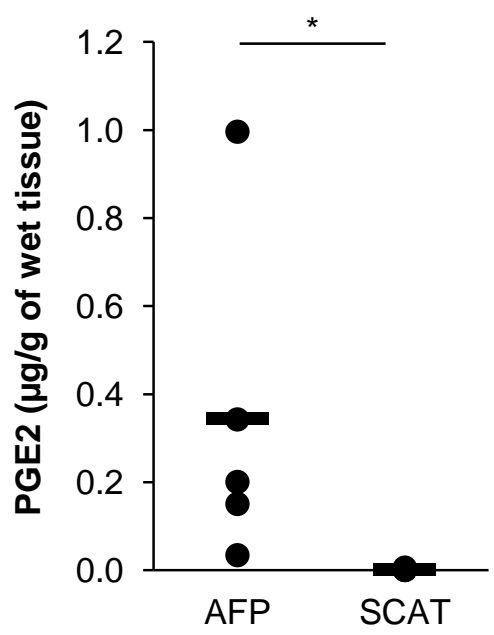

Figure 3 
A

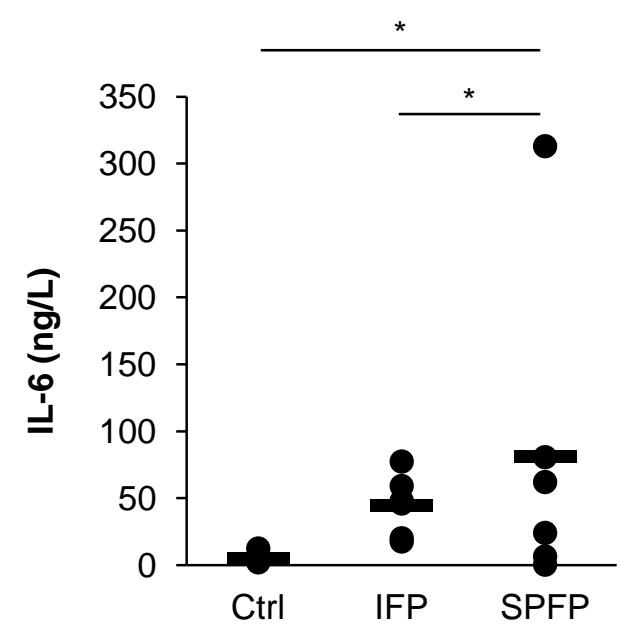

B

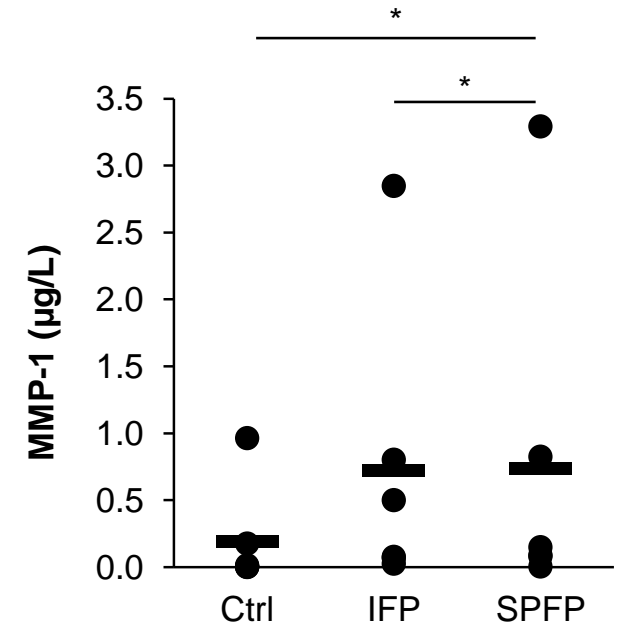

Figure 4 


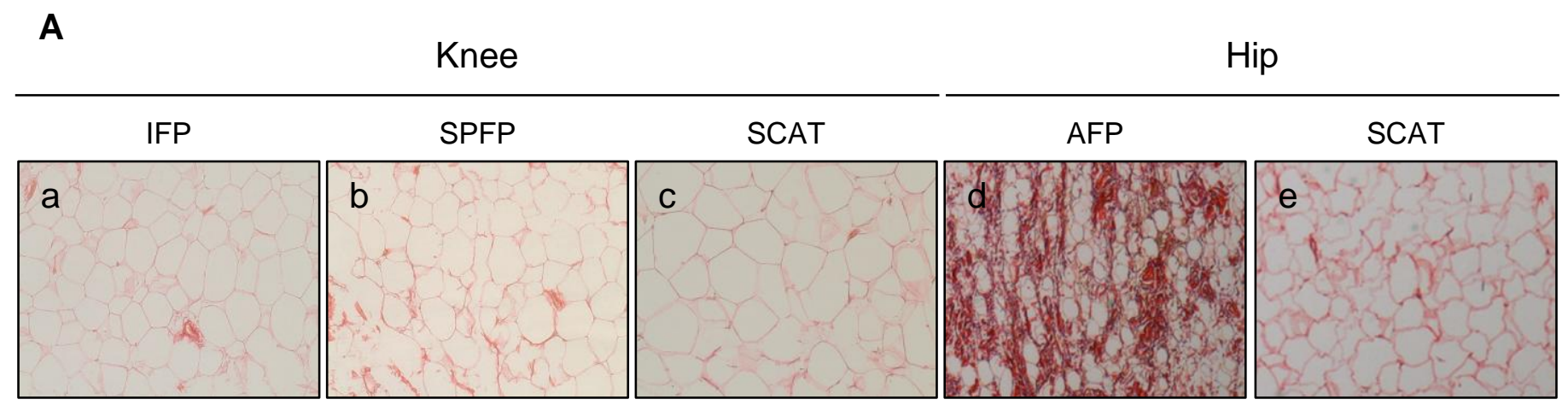

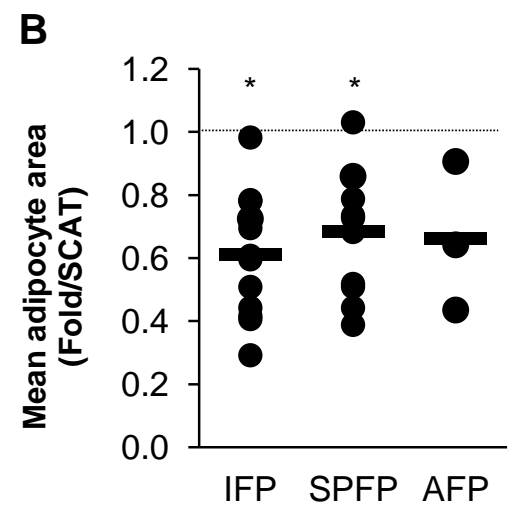

Figure 5 

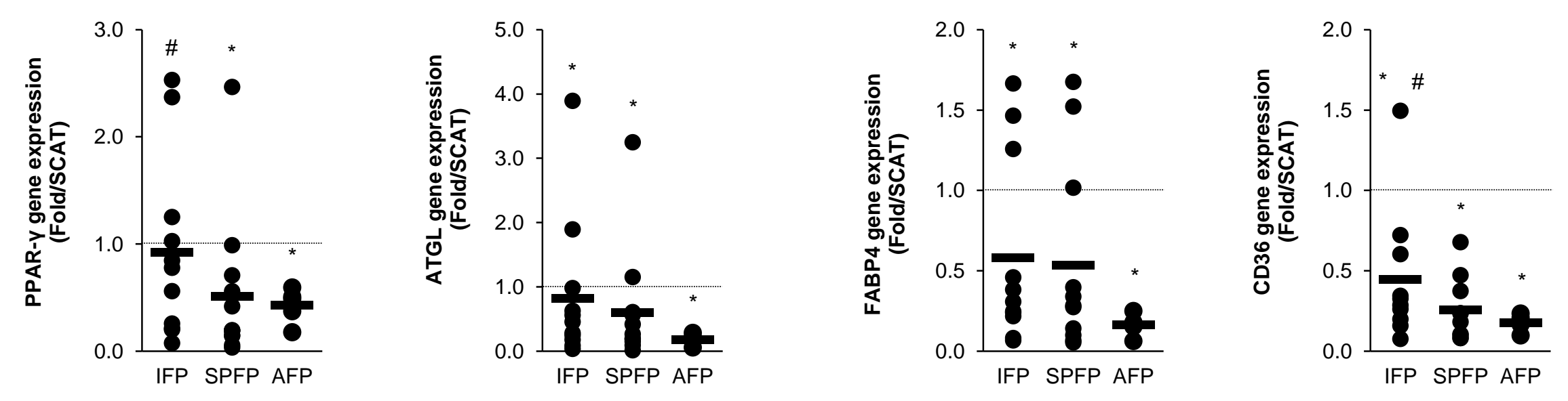

E

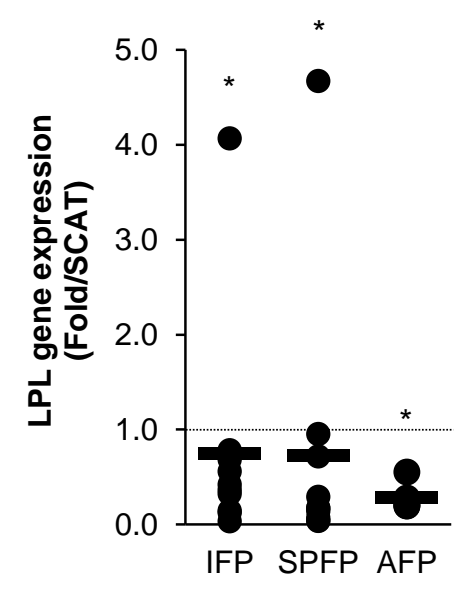

F

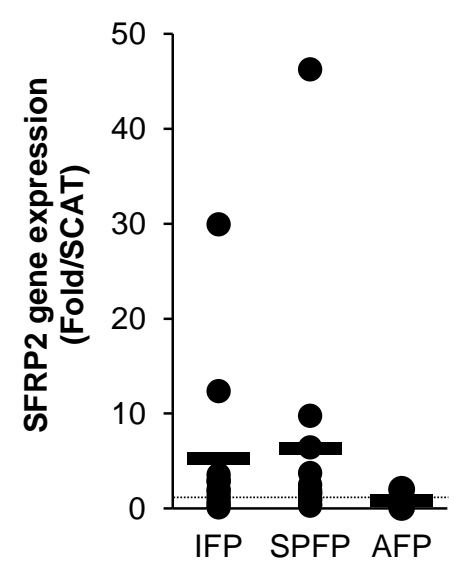

G

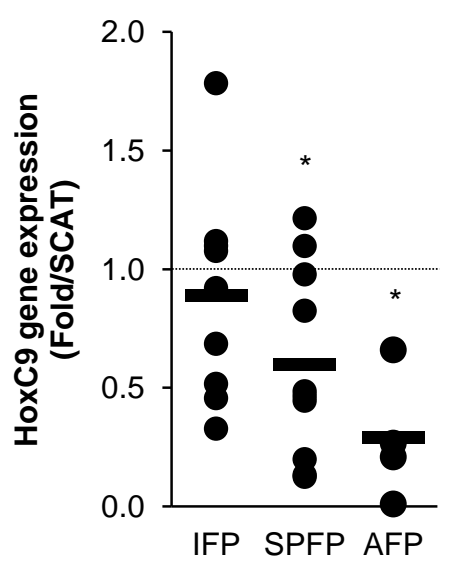

H

I
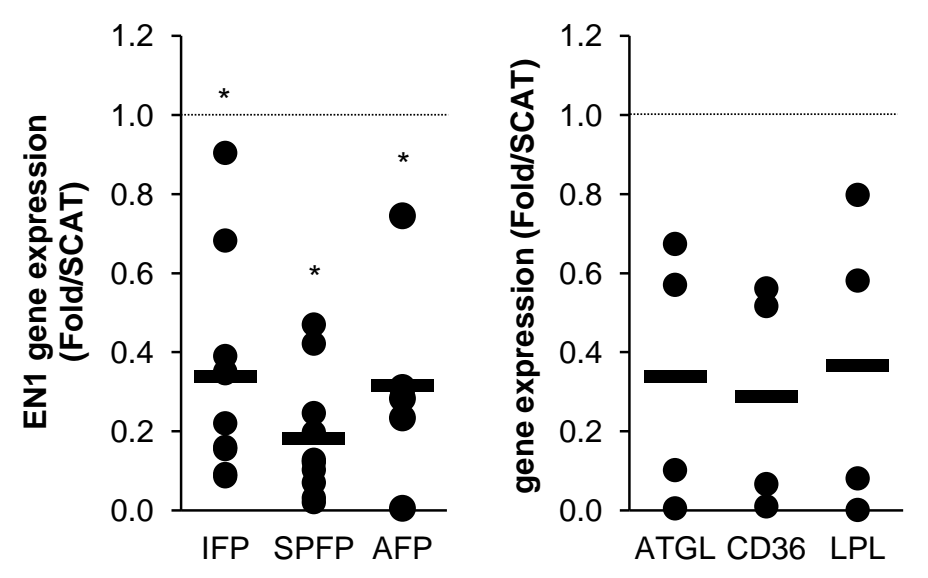

Figure 6 
A

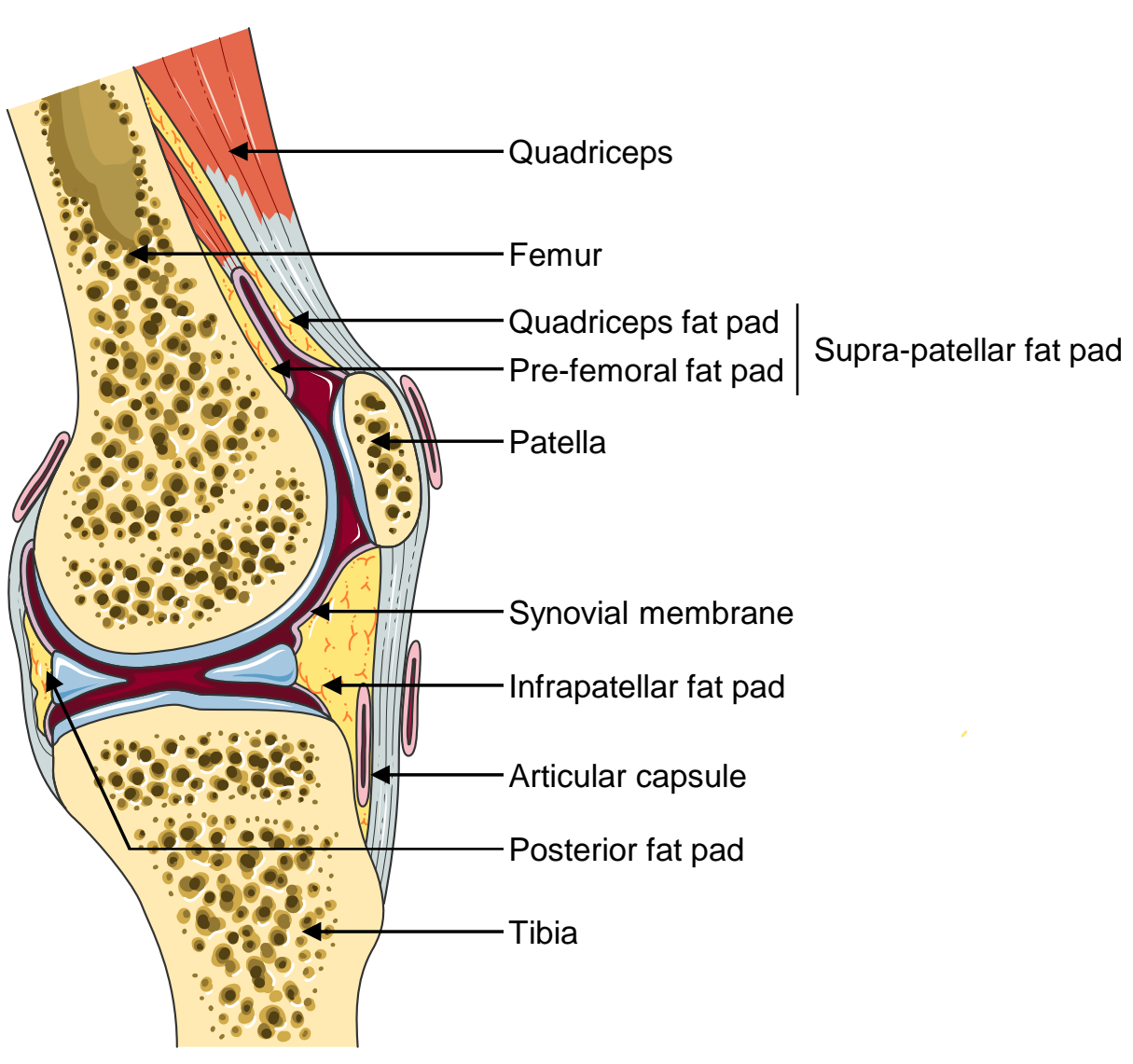

B

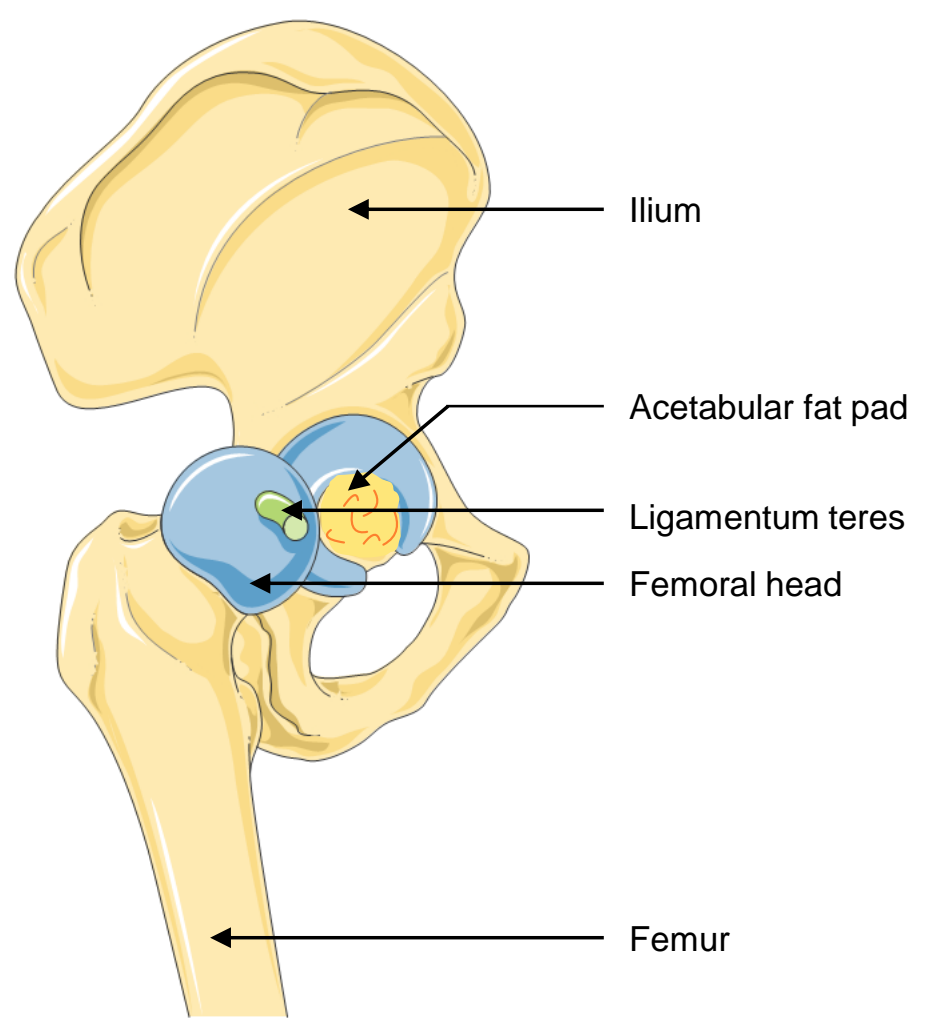




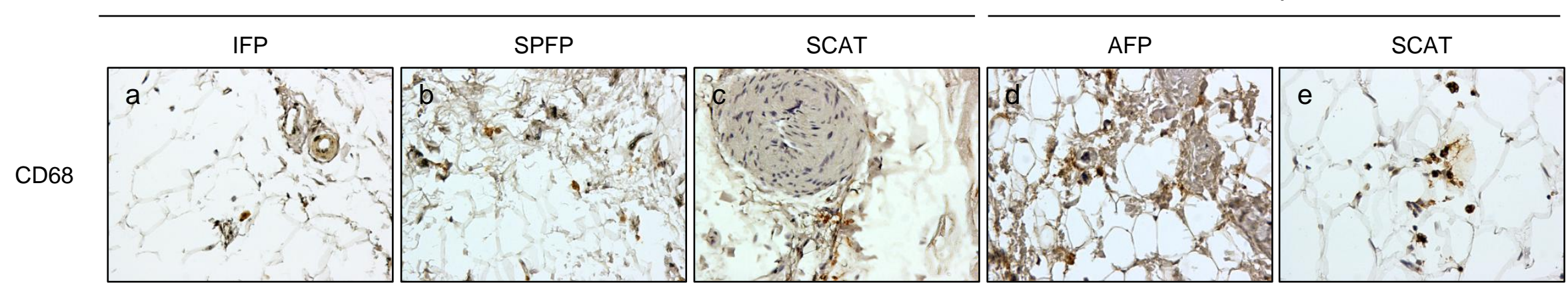

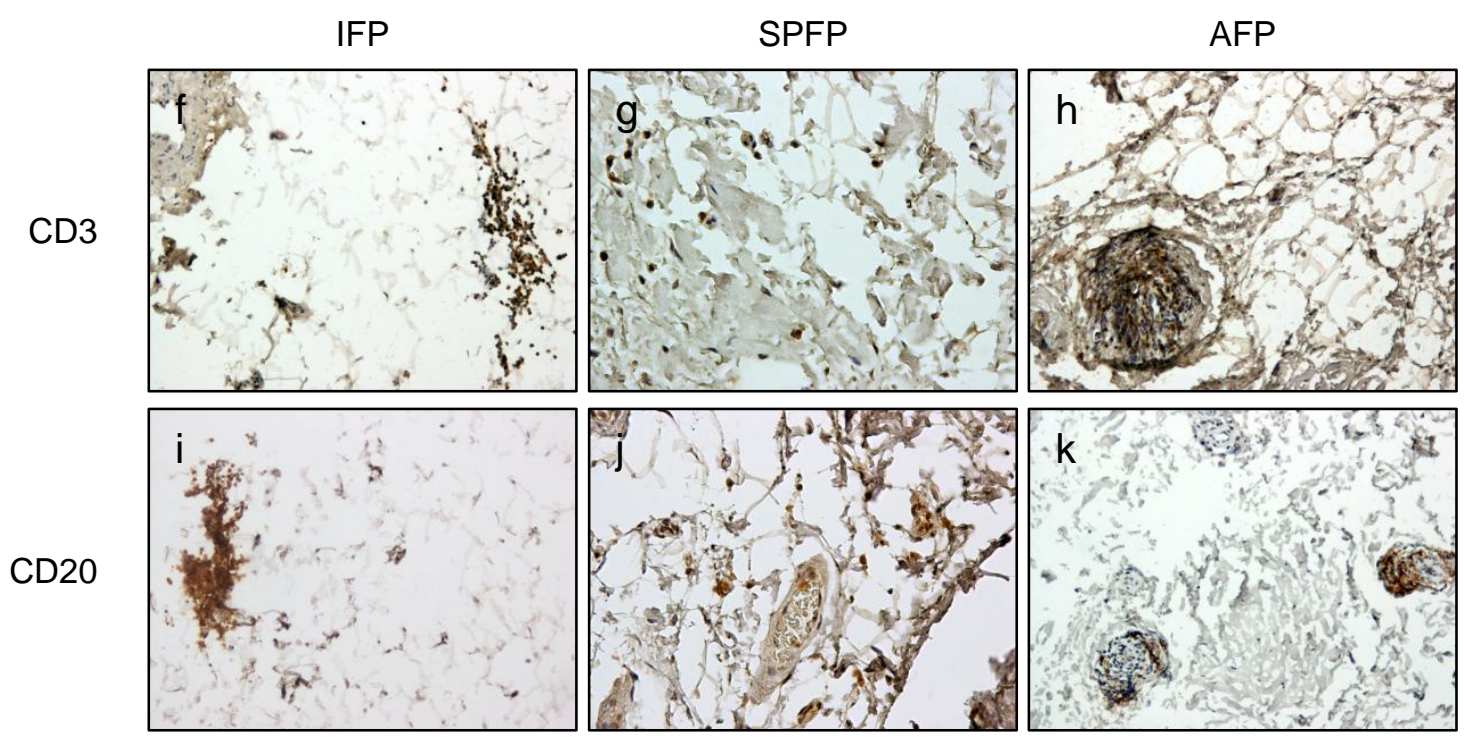



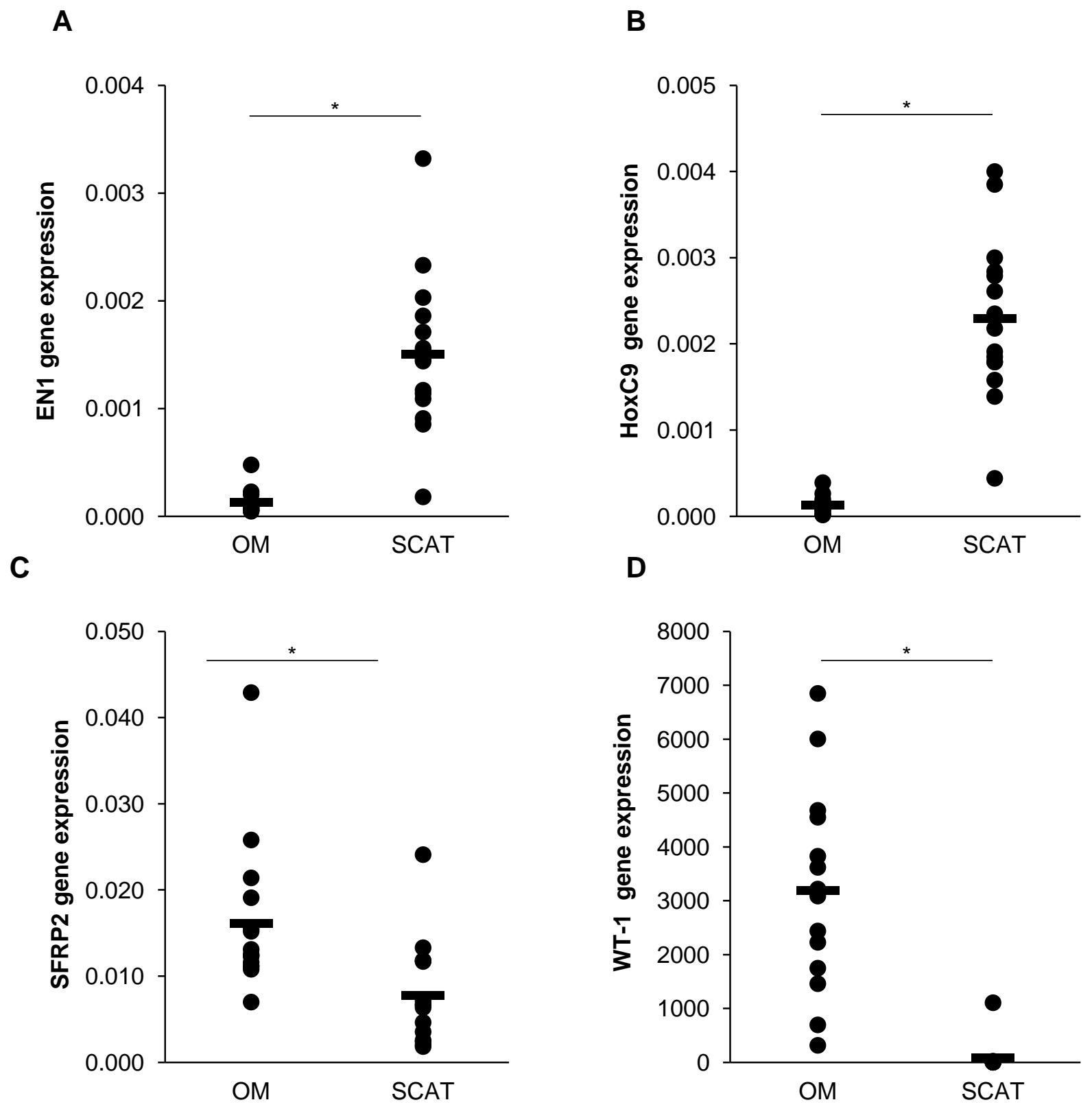

Supplementary Figure 3 
Supplementary Table 1. Sequence of primers used for RT-PCR studies.

\begin{tabular}{lll}
\hline Gene & Forward primer (5' $\mathbf{3}$ ') & Reverse primer (5'-3') \\
\hline 18s & GCAATTATTCCCCATGAACG & GGGACTTAATCAACGCAAGC \\
\hline PPAR- $\gamma$ & GACAGGAAAGACAACAGACAAATC & GGGGTGATGTGTTTGAACTTG \\
\hline ATGL & CCAAGCGGAGGATTACTCG & GAGAGGGTGGTCAGCAGGT \\
\hline FABP4 & CCTTTAAAAATACTGAGATTTCCTTCA & GGACACCCCCATCTAAGGTT \\
\hline LPL & TGACCAATAAGAAAGTCAACAGAATTA & CTCTGGTGAATGTGTGTAAGACG \\
\hline CD36 & GTGCCTATTCTTTGGCTTAATGA & TTACTTGACTTCTGAACATGTTTGC \\
\hline EN1 & TCGGACAGGTGCTATCGAA & AAGGAGTTCGCAGTTTCGTC \\
\hline HoxC9 & CGTGCCCTCTCAGTCGTC & GCCAAGTCCGCATGTAGC \\
\hline sFRP2 & GCCTCGATGACCTAGACGAG & GGTCGCACTCAAGCATGTC \\
\hline Wt1 & AGCTCAAAAGACACCAAAGGAG & GGGAGAACTTTCGCTGACAA \\
\hline
\end{tabular}


Supplementary Table 2. Characteristics of knee and hip OA patients.

\begin{tabular}{lrr}
\hline & Knee & Hip \\
\hline Number of patients & 38 & 5 \\
Age, years & $73.3 \pm 6.5$ & $73.0 \pm 3.3$ \\
Gender (Female), n (\%) & $29(76.3)$ & $3(60)$ \\
Weight, kg & $76.7 \pm 12.4$ & $75.2 \pm 11.7$ \\
Height, cm & $161.7 \pm 7.6$ & $165.6 \pm 7.2$ \\
Body mass index, kg/m ${ }^{2}$ & $29.3 \pm 5.0$ & $27.4 \pm 4.4$ \\
Hypertension, $\mathrm{n}(\%)$ & $29(76.3)$ & $3(60)$ \\
Diabetes, $\mathrm{n}(\%)$ & $5(13.2)$ & $1(20)$ \\
Dyslipidemia, $\mathrm{n}(\%)$ & $18(47.4)$ & $2(40)$ \\
Metabolic syndrome, $\mathrm{n}(\%)$ & $16(42.1)$ & $2(40)$ \\
\hline
\end{tabular}


Supplementary Table 3. Relationship between adipose tissue characteristics and obesity and gender

\begin{tabular}{|c|c|c|c|c|c|c|c|}
\hline & & \multicolumn{3}{|c|}{ OBESITY } & \multicolumn{3}{|c|}{ GENDER } \\
\hline & & Obese & Non-obese & p-value & Female & Male & p-value \\
\hline \multirow{6}{*}{$\hat{\theta}$} & IL-6 (ng/g) & $12.6[2.2-23.0]$ & $66.3[16.7-115.8]$ & 0.06 & $26.8[6.9-46.7]$ & $69.5[0.0-153.5]$ & 0.46 \\
\hline & IL-8 (ng/g) & $16.0[6.4-25.5]$ & $30.3[2.9-57.8]$ & 0.65 & $18.8[9.6-28.0]$ & $33.8[0.0-79.0]$ & 0.93 \\
\hline & $\mathrm{PGE}_{2}(\mathrm{ng} / \mathrm{g})$ & $36.7[0.0-79.3]$ & $65.5[0.0-133.4]$ & 0.57 & $45.2[8.1-82.2]$ & $66.4[0.0-172.6]$ & 0.54 \\
\hline & Fibrosis (\%) & $37.9[13.6-62.2]$ & $27.5[16.7-38.2]$ & 0.50 & $27.3[14.1-40.5]$ & $43.8[34.5-53.2]$ & 0.16 \\
\hline & Vascular area (\%) & $4.1[2.4-5.7]$ & $4.0[2.5-5.5]$ & 0.66 & $3.3[2.4-4.3]$ & $6.5[3.4-9.7]$ & $\underline{0.04}$ \\
\hline & Adipocyte area (pixels) & $0.16[0.12-0.19]$ & $0.16[0.14-0.19]$ & 0.56 & $0.15[0.13-0.17]$ & $0.22[0.22-0.22]$ & 0.11 \\
\hline \multirow{6}{*}{$\frac{\hat{r}}{\sqrt{n}}$} & IL-6 (ng/g) & $37.7[0.0-79.3]$ & $79.7[27.0-132.3]$ & 0.10 & $50.6[8.8-92.5]$ & $77.0[23.0-131.1]$ & 0.16 \\
\hline & IL-8 (ng/g) & $40.4[0.0-81.6]$ & $45.1[12.0-78.1]$ & 0.63 & $40.4[5.1-75.6]$ & $48.8[23.7-74.0]$ & 0.13 \\
\hline & $\mathrm{PGE}_{2}(\mathrm{ng} / \mathrm{g})$ & $152.2[17.8-286.5]$ & $99.0[0.0-224.9]$ & 0.83 & $146.6[24.9-268.3]$ & $70.9[0.0-142.4]$ & 0.59 \\
\hline & Fibrosis (\%) & $22.0[14.3-29.6]$ & $29.3[22.7-36.0]$ & 0.09 & $25.6[19.7-31.5]$ & $35.8[21.0-50.6]$ & 0.35 \\
\hline & Vascular area (\%) & $3.8[1.6-6.0]$ & $4.1[2.4-5.7]$ & 0.64 & $3.9[2.8-4.9]$ & $5.6[0.0-13.9]$ & 1.0 \\
\hline & Adipocyte area (pixels) & $0.22[0.18-0.25]$ & $0.16[0.14-0.18]$ & 0.06 & $0.18[0.15-0.21]$ & $0.20[0.20-0.20]$ & 0.60 \\
\hline \multirow{6}{*}{$\underset{\sim}{\mathcal{E}}$} & IL-6 (ng/g) & $6.9[0.0-15.5]$ & $12.0[3.7-20.3]$ & 0.19 & $7.2[0.9-13.6]$ & $15.0[0.8-29.3]$ & 0.14 \\
\hline & IL-8 (ng/g) & $7.1[0.5-13.7]$ & $5.7[3.4-8.1]$ & 0.57 & $6.6[2.0-11.3]$ & $5.9[2.8-8.9]$ & 0.46 \\
\hline & $\mathrm{PGE}_{2}(\mathrm{ng} / \mathrm{g})$ & $0.4[0.3-0.5]$ & $0.9[0.2-1.7]$ & 0.22 & $0.3[0.3-0.4]$ & $1.4[0.2-2.5]$ & $\leq 0.001$ \\
\hline & Fibrosis (\%) & $18.4[9.7-27.1]$ & $19.1[12.9-25.3]$ & 1.0 & $18.9[13.9-23.9]$ & $21.5[0.0-44.7]$ & 0.81 \\
\hline & Vascular area (\%) & $1.5[1.0-2.0]$ & $1.4[1.0-1.9]$ & 1.0 & $1.4[1.1-1.7]$ & $1.2[0.0-2.4]$ & 0.67 \\
\hline & Adipocyte area (pixels) & $0.27[0.23-0.32]$ & $0.27[0.24-0.30]$ & 0.77 & $0.27[0.24-0.30]$ & $0.29[0.29-0.29]$ & 0.88 \\
\hline
\end{tabular}

